\title{
Systematic review and meta-analysis of the 2010 ASGE non-invasive predictors of choledocholithiasis and comparison to the 2019 ASGE predictors
}

\author{
Louie Wang $^{1} \oplus \cdot$ Sarah Mirzaie $^{1} \cdot$ Tavit Dunnsiri $^{2} \cdot$ Formosa Chen $^{1,4} \cdot$ Holly Wilhalme $^{6} \cdot \operatorname{lan}$ T. MacQueen ${ }^{1,3}$. \\ Henry Cryer $^{1,3}$ • Anaar Eastoak-Siletz ${ }^{1,3}$ • Michelle Guan ${ }^{1}$. Callie Cuff ${ }^{1}$. James H. Tabibian ${ }^{1,5}$
}

Received: 14 October 2021 / Accepted: 10 December 2021 / Published online: 24 January 2022

(c) The Author(s) 2022

\begin{abstract}
In 2019, the American Society for Gastrointestinal Endoscopy (ASGE) guideline on the endoscopic management of choledocholithiasis modified the individual predictors of choledocholithiasis proposed in the widely referenced 2010 guideline to improve predictive performance. Nevertheless, the primary literature, especially for the 2019 iteration, is limited. We performed a systematic review with meta-analysis to examine the diagnostic performance of the 2010, and where possible the 2019, predictors. PROSPERO protocol CRD42020194226. A comprehensive literature search from 2001 to 2020 was performed to identify studies on the diagnostic performance of any of the 2010 and 2019 ASGE choledocholithiasis predictors. Identified studies underwent keyword screening, abstract review, and full-text review. The primary outcomes included multivariate odds ratios (ORs) and 95\% confidence intervals for each criterion. Secondary outcomes were reported sensitivities, specificities, and positive and negative predictive value. A total of 20 studies met inclusion criteria. Based on reported ORs, of the 2010 guideline "very strong" predictors, ultrasound with stone had the strongest performance. Of the "strong" predictors, CBD $>6 \mathrm{~mm}$ demonstrated the strongest performance. "Moderate" predictors had inconsistent and/or weak performance; moreover, all studies reported gallstone pancreatitis as non-predictive of choledocholithiasis. Only one study examined the new predictor (bilirubin $>4 \mathrm{mg} / \mathrm{dL}$ and $\mathrm{CBD}>6 \mathrm{~mm}$ ) proposed in the 2019 guideline. Based on this review, aside from CBD stone on ultrasound, there is discordance between the proposed strength of 2010 choledocholithiasis predictors and their published diagnostic performance. The 2019 guideline appears to do away with the weakest 2010 predictors.
\end{abstract}

Keywords American Society for Gastrointestinal Endoscopy · Choledocholithiasis · Endoscopic retrograde cholangiopancreatography $\cdot$ ERCP $\cdot$ Cholelithiasis

Louie Wang

gngnlouie@gmail.com

1 David Geffen School of Medicine, University of California, 10833 Le Conte Ave, Los Angeles, CA 90095, USA

2 Department of Internal Medicine, University of California, Los Angeles, CA, USA

3 Department of Surgery, University of California, Los Angeles, CA, USA

4 Division of Gastroenterology, Department of Surgery, Olive View-UCLA Medical Center, Sylmar, CA, USA

5 Division of Gastroenterology, Department of Medicine, Olive View-UCLA Medical Center, Sylmar, CA, USA

6 Statistics Core, Department of Medicine, University of California, Los Angeles, CA, USA

\section{Introduction}

Cholelithiasis affects over 25 million individuals in the United States (USA), [1, 2], with associated healthcare expenditures approaching \$10 billion annually [3]. Approximately $10-20 \%$ of patients with cholelithiasis also have choledocholithiasis (CDL) [4, 5]. Complications of CDL, in turn, include acute pancreatitis (AP) and cholangitis (AC), among others [6]. These conditions increase morbidity and healthcare expenditures, highlighting the importance of early and accurate diagnosis.

Endoscopic retrograde cholangiopancreatography (ERCP) is considered a gold standard diagnostic and a therapeutic modality for choledocholithiasis [7, 8]. However, it carries risk of serious adverse events and should thus be performed selectively, based on pre-test probability 
[9-11]. In 2010, the American Society for Gastrointestinal Endoscopy (ASGE) guideline on the evaluation of CDL [12] proposed common bile duct (CBD) stone on abdominal ultrasound (US) (US with stone), AC, and total bilirubin (Tbili) $>4 \mathrm{mg} / \mathrm{dL}$ as "very strong" predictors;" Tbili 1.8-4 mg/dL and CBD dilation (> $6 \mathrm{~mm}$ ) as "strong" predictors;" and abnormal liver function test (ALFT), age $>55$, and AP as "moderate" predictors." Likelihood of CDL for any very strong predictor is high, presence of both strong predictor is high, while likelihood of CDL for a single strong predictor or any moderate predictor(s) was not discussed. ERCP was recommend in patients with high likelihood of CDL. Since the publication of this guideline, studies worldwide have examined the performance of these predictors in different patient populations; in some instances, performance has been marginal [13, 14]. In 2019, the ASGE published an updated CDL guideline [15], in which AC, stone on imaging, and the combination of Tbili $>4 \mathrm{mg} / \mathrm{dL}+\mathrm{CBD}$ dilation were proposed as "high-probability" predictors and ALFT, age $>55$, or CBD dilation (without Tbili $>4 \mathrm{mg} / \mathrm{dL}$ ) as "intermediate." AP and Tbili $1.8-4 \mathrm{mg} / \mathrm{dL}$ were removed from the predictor list, and there were no longer "moderate" strength/probability predictors. In this update, predictors with intermediate probability was recommended additional imaging studies, while predictors with high probability is recommended ERCP, unchanged from the 2010 guideline.

The predictors in the 2019 CDL guideline were modified based on data from five studies examining the performance of the 2010 predictors. For example, the combined predictor "Tbili $>4 \mathrm{mg} / \mathrm{dL}+\mathrm{CBD}>6 \mathrm{~mm}$ " was, for example, recommended given the improved specificity in three studies $[13,16,17]$, and AP was removed due to "lack of correlation" [15]. Tbili $>4 \mathrm{mg} / \mathrm{dL}$ and $1.8-4 \mathrm{mg} /$ $\mathrm{dL}$ were removed for uncertain reasons. Over the past decade, however, there have been additional studies examining the 2010 predictors [13, 16-19], and thus the evidence to support the 2019 modifications may in fact be greater.

The goal of this systematic review is to examine the diagnostic performance of the 2010 ASGE guideline CDL predictors as reported by worldwide studies, and compare the reviewed results with the changes proposed in the newly published 2019 ASGE guideline. This project was not originally designed to involve the need of a meta-analysis. However, an attempt is made to quantify the reporting primary and secondary outcomes in a standardized fashion. We hope that a statistical standardization of primary outcomes will bring further clarity when comparing the predictors' clinical performances. We believe a more thorough characterization of predictors' performances in different patient populations can better allow clinicians to apply these guidelines and formulate future modifications and risk-stratification criteria.

\section{Methods}

\section{Search strategy}

Following a methodological process of assessing the literatures, PRISMA 2020 Checklist was used to establish a clear guideline involve both the inclusion and exclusion criteria. The literature review involved a comprehensive search of PubMed, EMBase, SCOPUS, and WEB of SCIENCE for studies published from 2000 to 2020, conducted in adult populations, and written in English (PROSPERO CRD42020194226). In brief, search terms included: choledocholithiasis, cholelithiasis, endoscopic retrograde cholangiopancreatography, ERCP, ASGE, endoscopic US or endosonography, biochemical parameter, CBD, and statistics. Search results were extracted and organized using Zotero 5.0 for further keyword screening after review of duplicates. Two independent reviewers (LW \& SM) screened the title and abstracts of the studies identified in the preliminary search were screened, and studies that assessed the diagnostic performance of any ASGE choledocholithiasis predictor was reviewed in full. individually reviewed the abstracts of the screened-in studies to assess the relevance to the study objective. Studies that led to disagreement or uncertainty between the two reviewers were adjudicated by a third reviewer (JT). Afterward, fulltext of the included studies was obtained. Full-text reviews were then conducted based on selection criteria alongside data extraction.

The exact search for PubMed database was conducted using: ("Cholelithiasis"[Mesh] OR "Choledocholithiasis"[Mesh] OR "Gallbladder Diseases"[Mesh] OR "Cholecystitis"[Mesh] OR "Cholecystolithiasis"[Mesh]) AND (ercp[MeSH Terms] OR (intraoperative_cholangiogram) OR Endosonography[MeSH Terms] OR Cholangiopancreatography, Magnetic Resonance[Mesh]) AND ((Risk calculator) OR algorithm[MeSH Terms] OR Predictive Value of Tests[MeSH Terms] OR (Predictive) OR (Prognostic Value) OR Quality of Health Care[MeSH Terms]) Filters: Humans, English, Adult: 19+ years, from 2000 to 2020.

The exact search for EMBASE database was conducted using: ('Cholelithiasis' OR 'common bile duct stone' OR 'gallbladder disease' OR 'cholecystitis' OR 'cholelithiasis') AND ('endoscopic retrograde cholangiopancreatography' OR 'cholangiography' OR 'endoscopic ultrasonography' $O R$ 'magnetic resonance cholangiopancreatography') AND ('health care quality' OR 'prognostic value' OR (predictive) OR 'predictive value' OR 'algorithm' OR 'risk calculator') AND [adult]/lim AND [humans]/lim AND [2000-2020]/py.

The exact search for WEB of SCIENCE database was conducted using: 
$T S=($ Cholelithiasis OR Choledocholithiasis OR Gallbladder Diseases OR Cholecystitis OR Cholecystolithiasis) AND (ERCP OR intraoperative_cholangiogram OR Endoscopic ultrasound OR MRCP) AND (Risk calculator OR algorithm OR Predictive OR Prognostic Value) AND LANGUAGE: (English) Refined by: [excluding] RESEARCH AREAS: (PEDIATRICS).

\section{Selection criteria}

We included original studies that reported statistical findings regarding the predictive value of ASGE-listed predictors in patients with or suspected CDL. Exclusion criteria were as follows:

- Studies that did not include 'predict' and 'associat in the title or abstract

- Studies published due to abstract-only nature, or full-text link not found on databases.

- Studies lacking statistical values for the ASGE predictors and/or focusing on a different outcome, such as the longterm impact of endoscopic CDL management.

- Studies focused on a specific demographic population (pregnancy, elderly, or immunocompromised, etc.)

\section{Data extraction}

The data extraction proceeded with standardized guidelines, focusing on reported statistical quantification of predictor: ORs, univariate vs. multivariate OR analyses, upper and lower $95 \%$ confidence interval (CI), $p$ values, sensitivity, specificity, PPV, and NPV. The sample size associated with each reported predictor was also extracted.

For ALFT, only studies that literally stated or reported "abnormal liver function tests per ASGE" (or similar) were extracted. Data related to the predictor "US with stone" were extracted if the reporting studies specified procedure as transabdominal US. Tbili levels were reported in a standardized unit of $\mathrm{mg} / \mathrm{dL}$. The sample size mean age for the CBD $>6 \mathrm{~mm}$ predictor was extracted concerning confounding as CBD dilate associates with aging. Non ASGE predictors were excluded in the extraction. Only the initial set of clinical data, such as lab values, were extracted in cases of multiple sets of data being reported in a study.

\section{Data analysis}

Formulation of conversation are drafted according to the Cochran Handbook [20]. For each predictor in either multivariate or univariate analysis, forest plots were generated from Prism- $\mathrm{GraphPad}^{\circledR}$ with a calculated inverse-variance weight (1/Variance). Variance was calculated using the following formula: $\frac{1}{\text { Weight }}=\left(\frac{(\ln (\text { upper 95\% CI })-\ln (\text { lower } 95 \% \mathrm{CI}))}{2 * 1.96}\right)^{2} \times \mathrm{n}$

where $n$ is the specific sample size reported a study for the specific predictor.

A weighted average OR (WaOR) was then calculated using the reported ORs of the predictors and their calculated inverse-variance weight by the following formula:

$\mathrm{Wa}$ OR $=\frac{\text { sum of }(\mathrm{OR} \times \text { weight })}{\text { sum of weight }}$

Although our review has grouped the predictor AP and $\mathrm{AC}$ on forest plots, studies had an inconstant definition for $\mathrm{AP}$ and $\mathrm{AC}$, which are labeled in results. The exact definition of AP and AC from studies are reported in the results.

When examining the predictive value of a criteria, we sought to report OR as the primary outcome. OR exceeding 1 indicates high strength of association between the predictor and CDL. Although OR is not meant for establishing a causation relationship, its strength of association fits our clinical definition of high predictive value in clinical management. Traditionally, ORs are also the calculation standard in statistical analysis. Thus, our review defined a predictor with a high WaOR to have high predictive strength.

Sensitivity, specificity, PPV, and NPV were also extracted as secondary outcomes. If a predictor had high specificity, it then demonstrated a high strength in ruling out the true negatives, thus helping in ruling in the true positives. Similarly, if a predictor has a high PPV, it then demonstrates a strong strength in ruling in the true positives from the total positives. Statistically, if a predictor has a strong predictive value, it shall be reported with high specificity and PPV.

\section{Results}

\section{Extraction results}

A total of 2242 studies were initially extracted. After excluding 382 duplicates, 1860 studies were identified for keyword screening. After excluding 1132 studies not containing "predict" or "associate" in the abstract, 728 studies were included for detailed abstract review conducted by two reviewers (LW and SM), with a third reviewer (JT) arbitrating. This process eliminated 600 studies, yielding 118 studies for full-text review. After full-text review, a total of 98 studies focusing on endpoints that were unrelated to the predictive value of ASGE predictors (41), did not report statistical data (25), had a population not primarily related to CDL (16), did not have complete data reported for extraction (8), or were reviews in nature (8) were excluded. In total 20 studies were included (Fig. 1). Aiming to improve internal validity, we followed the PRISMA 2020 


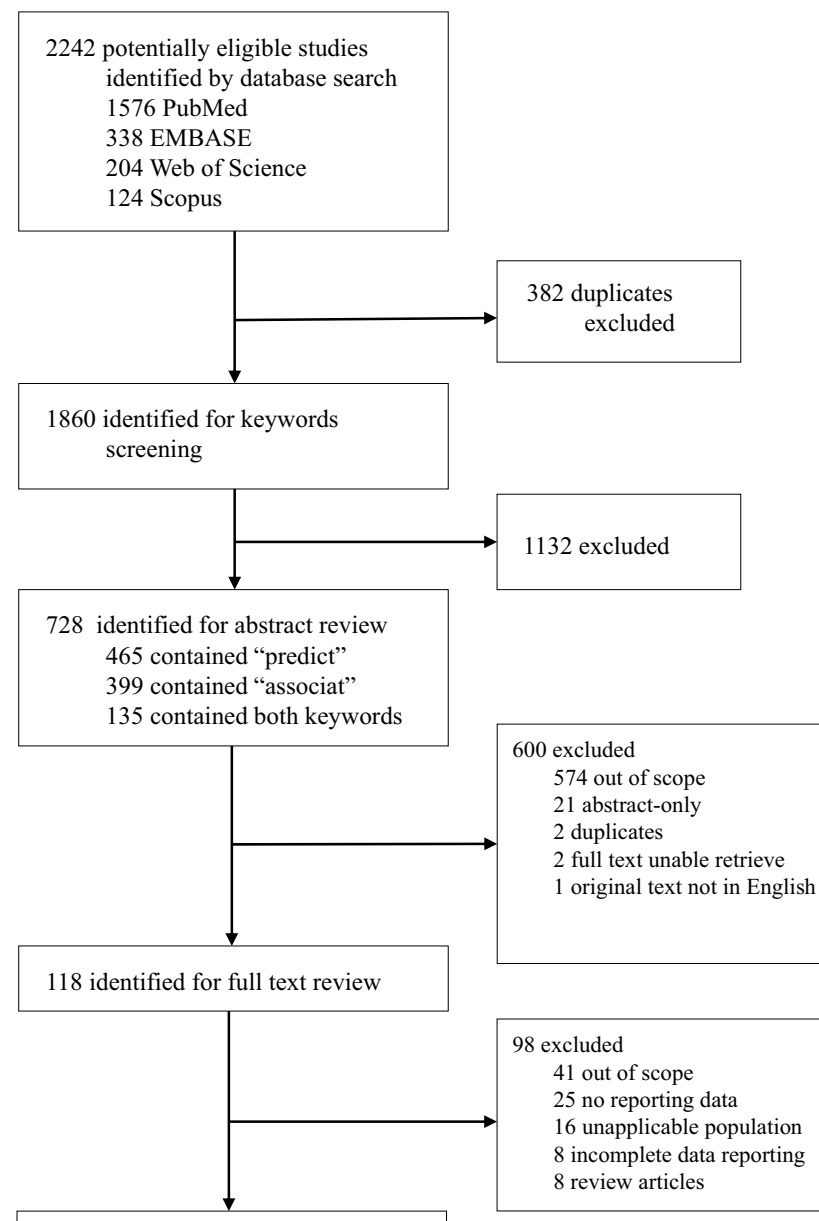

20 eligible studies

Fig. 1 A flowchart of the literature search, review, and selection process of this review

check-list to exclude all studies that met the exclusion criteria. The final 20 studies all met with the ordinally designed methodological quality. They had a median sample size of 165 , ranging between 18 and 2055. Three studies were published between 2000 and 2010, two between 2010 and 2015, and 15 after 2015. Among the primary outcomes, Suarez et al. is the only study reported ORs in multivariable instead of multivariate analysis. Regarding study types, 11 studies were reported as a retrospective and 9 as a prospective cohort. Eight studies were conducted in the United States (USA), two in South Korea and India each, and the studies remaining studies were individually from China, Lebanon, Lithuania, Portugal, Saudi Arabia, Spain, Sweden, and Turkey.

\section{Statistical findings}

\section{Overview of extraction results}

Of the 20 included studies that reported predictive statistical data for CDL, 13 reported ORs and their associated statistical data (95\% CI and $p$ values) for at least one ASGE CDL predictor. In these studies, six reported multivariateanalyzed ORs and six univariate-analyzed ORs. One study reported both multivariate- and univariate-analyzed ORs. A forest plot was generated for each ASGE predictor with studies that reported ORs using multivariate (Fig. 1) and univariate analysis (Fig. 2), and WaOR for each predictor in both multivariate and univariate analyses were calculated (Table 1). Detailed statistical findings regarding the OR-reported studies are presented in Figs. 2 and 3. Statistical details for Figs. 2 and 3 are reported in Tables 2 and 3, respectfully. All 20 studies reported sensitivity, specificity, PPV, and NPV for at least one ASGE predictor, as shown in Table 4. A meta-analysis for data reported in Table 4 was not attempted given concern for a lack of statistical significance data without reported range of confidence intervals, difficulty in attributing weights, and reporting bias. Detailed information regarding each included study were composed on Table 5.

\section{Stone found on abdominal ultrasound}

Five studies reported multivariate analyzed ORs with a WaOR of 8.62. Sample sizes ranged from 13 [24] to 524 [21]. He et al. [21] (OR 17.3; [95\% CI 12.6-23.8]) reported the highest OR, while Kuzu et al. [22] (OR 2.74; [95\% CI 1.63-4.60]) reported the lowest OR. Rubin et al. [19] (OR 6.65 ; [95\% CI 2.58-17.2]) carried the most inverse variance weight $(9.9 \%)$.

Three studies reported univariate analyzed ORs with a weighted average of 5.57. Sample sizes ranged from 44 [14] to137 [28]. Magalhaes et al. [18] (OR, 11.3; [95\% CI 5.32-23.8]) reported the highest OR, while Nárvaez Rivera et al. [14] (OR, 3.09; [95\% CI 1.45-6.58]) reported the lowest OR and carried the most inverse-variance weight (15\%) among these three studies.

Eleven studies reported sensitivity (mean 37\%, range 13-62\%), specificity (mean 94\%, range $85-100 \%$ ), PPV (mean 81\%, range 58-98\%), and NPV (mean 62\%, range 26-95\%) for this predictor.

\section{Acute cholangitis}

Five studies reported multivariate analyzed ORs with a WaOR of 2.29. Sample sizes ranged from 23 [19] to 463 [21]. Jeon et al. [25] (OR 5.84; [95\% CI 1.23-27.8]) reported the highest OR, while He et al. [21] (OR 0.9; [95\% 


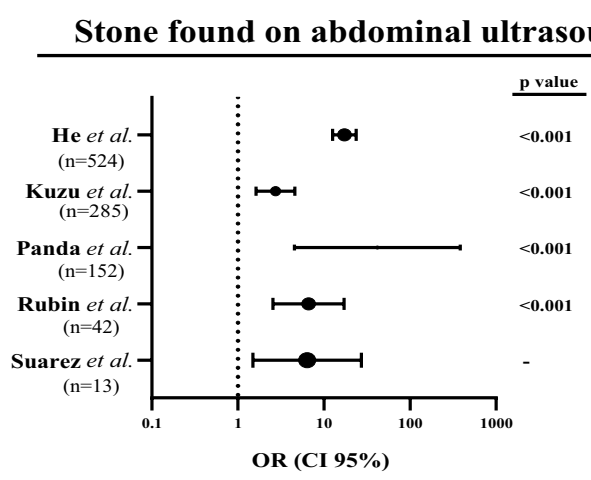

Total blirubin $>4 \mathrm{mg} / \mathrm{dL}$

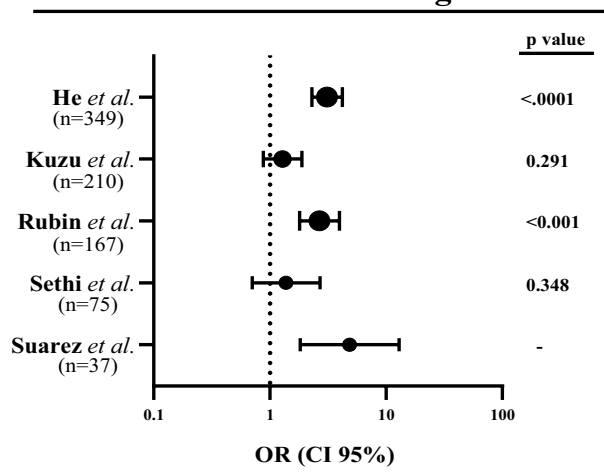

Common bile duct dilation $>6 \mathrm{~mm}$

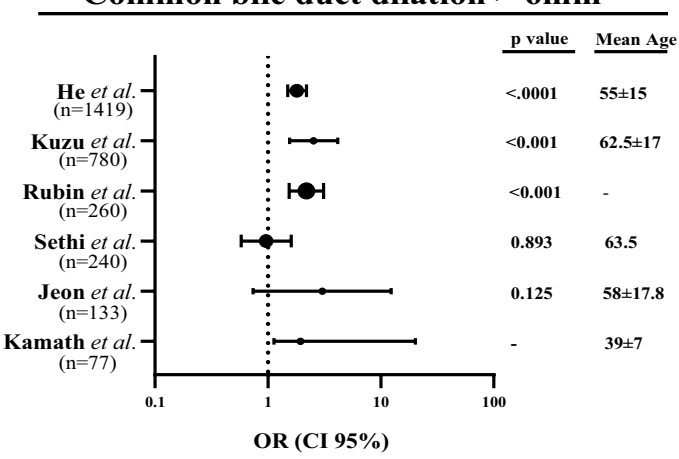

Abnormal liver function tests

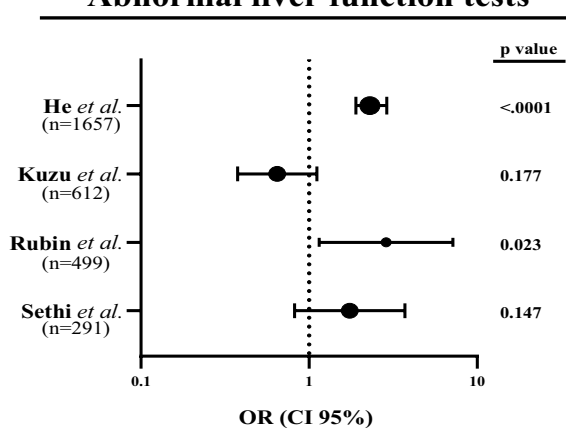

1 Cholangitis defined by 2013 Tokyo Guideline

2 Cholangitis defined by Charcot's triad

3 Cholangitis defined by upper abdominal pain, systemic infection, and abnormal liver function test markers

Fig. 2 Forest plots of studies with multivariate analyzed OR reporting

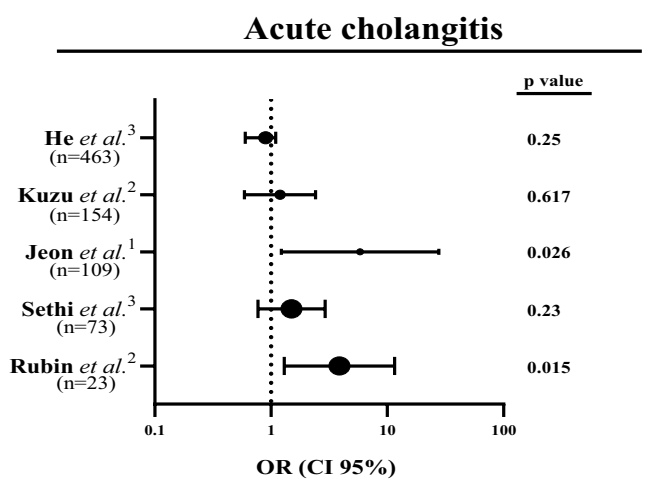

Total blirubin 1.8-4 mg/dL

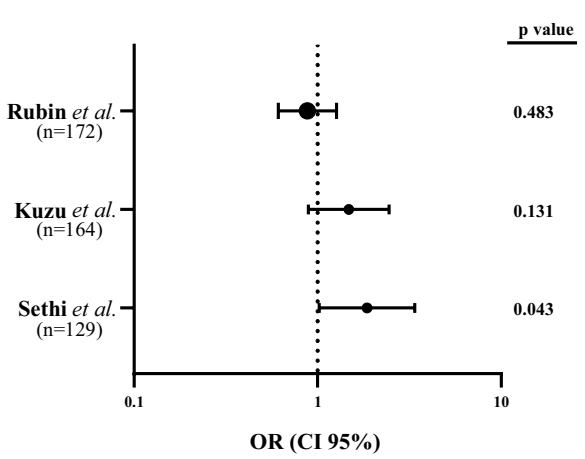

Age $>55$

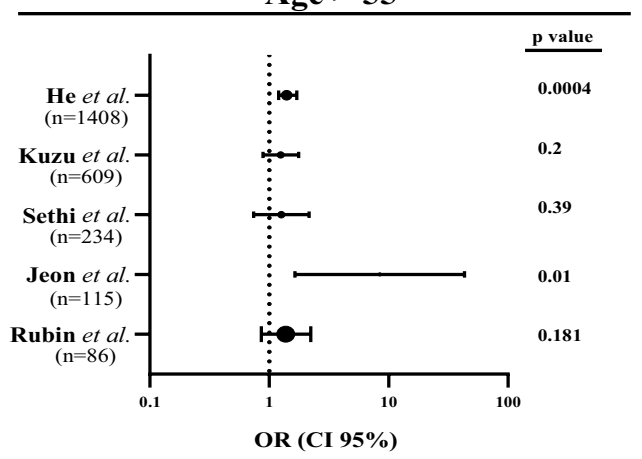

Acute pancreatitis

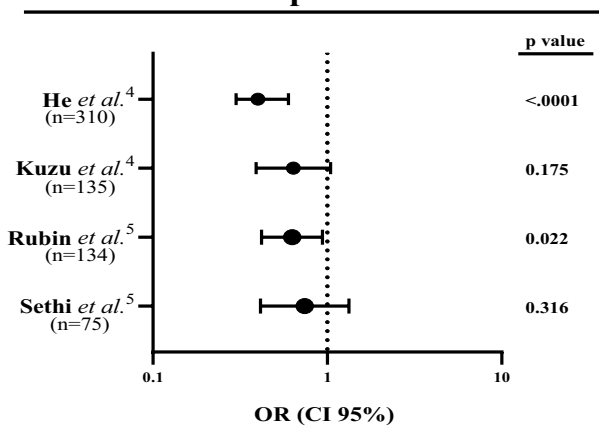

4 Pancreatitis defined by ICD 10 code code

5 Pancreatitis defined by adnominal pain, imagine evidence, and lipase elevated three-fold 
Table 1 Weighted average odds ratio for univariate and multivariate analyzed studies

\begin{tabular}{llll}
\hline 2010 ASGE definition & Predictors & $\begin{array}{l}\text { Univariate analysis average } \\
\text { weighted OR (WaOR) }\end{array}$ & $\begin{array}{l}\text { Multivariate analy- } \\
\text { sis average weighted } \\
\text { OR (WaOR) }\end{array}$ \\
\hline Very strong predictor & US with stone & 5.566 & 8.618 \\
& Acute Cholangitis & 2.500 & 2.290 \\
& Tbli $>4 \mathrm{mg} / \mathrm{dL}$ & 1.417 & 2.619 \\
Strong predictor & Tbli $1.8-4 \mathrm{mg} / \mathrm{dL}$ & 1.458 & 1.278 \\
& CBD $>6 \mathrm{~mm}$ & 3.697 & 1.900 \\
Moderate predictor & ALFT & 1.540 & 1.896 \\
& Age $>55$ & 1.742 & 1.567 \\
& Acute Pancreatitis & 0.811 & 0.620 \\
\hline
\end{tabular}

Sample sizes reported were relevant sample sizes, as not all studies had samples comprised entirely of (suspected) CDL patients
CI 0.6-1.1]) reported the lowest OR. Rubin et al. [19] (OR 3.88; [95\% CI 1.3-11.6]) carried the most inverse-variance weight $(14 \%)$.

Five studies reported univariate analyzed ORs with a WaOR of 2.5. Sample sizes ranged from 73 [26] to 184 [29]. Magalhaes et al. [18] (OR 6.48; [95\% CI 1.93-21.8]) reported highest OR, while $\mathrm{He}$ et al. [21] (OR 1.09; [95\% CI 0.76-1.55]) reported the lowest OR. Lee et al. [29] (OR 1.09; [95\% CI 0.76-1.55]) carried the most inverse-variance weight (16\%) among these three studies.

Nine studies reported sensitivity (mean $23 \%$, range $18-32 \%$ ), specificity (mean $89 \%$, range $70-100 \%$ ), PPV (mean 71\%, range 9-100\%), and NPV (mean 56\%, range $22-88 \%)$ for this predictor.

\section{Total bilirubin}

Tbili $>4 \mathrm{mg} / \mathrm{dL}$ Five studies reported multivariate analyzed ORs with a WaOR of 2.62. Sample sizes ranged from 37 [24] to 349 [21]. Suarez et al. [24] (OR 4.85; [95\% CI 1.8212.9]) reported the highest OR, while Kuzu et al. [22] (OR 1.29; [95\% CI 0.88-1.89]) reported the lowest OR. Rubin et al. [19] (OR 2.67; [95\% CI 1.8-3.97] carried the most inverse-variance weight (15\%).

Five studies reported univariate analyzed ORs with a WaOR of 1.42. Sample sizes ranged from 75 [26] to 366 [30]. Chisholm et al. [30] (OR 7.97; [95\% CI 3.06-20.8]) reported the highest OR, while Lee et al. [29] (OR 1.1; [95\% CI 0.72-1.67]) reported the lowest OR and carried the most inverse-variance weight (19\%) among these three studies.

Eight studies reported sensitivity (mean 35\%, range 20-61\%), specificity (mean 79\%, range 44-95\%), PPV (mean $71 \%$, range $57-93 \%$ ), and NPV (mean $47 \%$, range $22-65 \%)$ for this predictor.

Tbili $1.8-4 \mathrm{mg} / \mathrm{dL}$ Three studies reported multivariate analyzed ORs with a WaOR of 1.28. Sample sizes ranged from 129 [26] to 172 [19]. Sethi et al. [26] (OR, 1.86; [95\%
CI, 1.02-3.38]) reported the highest OR, while Rubin et al. [19] (OR, 0.88; [95\% CI, 0.61-1.27]) reported the lowest $\mathrm{OR}$ and carried the most inverse-variance weight (17\%).

Four studies reported univariate analyzed ORs with a WaOR of 1.46. Sample sizes ranged from 66 [14] to 158 [29]. Magalhaes et al. [18] (OR 3.15; [95\% CI 1.63-6.08]) reported the highest OR, while Lee et al. [29] (OR 0.86; [95\% CI 0.59-1.24]) reported the lowest OR. Nárvaez Rivera et al. [14] (OR 0.89; [95\% CI 0.5-1.56]) carried the most inverse-variance weight (18\%) among these three studies.

Five studies reported sensitivity (mean 33\%, range 20-61\%), specificity (mean $72 \%$, range $63-73 \%$ ), PPV (mean $65 \%$, range $54-84 \%$ ), and NPV (mean $42 \%$, range $43-53 \%)$ for this predictor.

\section{$\mathrm{CBD}>6 \mathrm{~mm}$}

Six studies reported multivariate analyzed ORs with a WaOR of 1.9. Sample sizes ranged from 77 [27] to 1419 [21]. Jeon et al. [25] (OR 3.02; [95\% CI 0.74-12.3]) reported the highest OR, while Sethi et al. [26] (OR 0.97; [95\% CI 0.58-1.61]) reported the lowest OR. Rubin et al. [19] (OR 2.19; [95\% CI 1.54- 3.12]) carried the most inverse-variance weight (12\%).

Six studies reported univariate analyzed ORs with a WaOR of 3.70. Sample sizes ranged from 193 [14] to 368[29]. Chisholm et al. [30] (OR 7.83; [95\% CI 3.87-15.8]) reported highest OR, while Sethi et al. [26] (OR 1; [95\% CI 0.63-1.59]) reported the lowest OR. Lee et al. [29] (OR 3.03; [95\% CI 2.12-4.3]) carried the most inverse-variance weight $(8.4 \%)$ among these three studies.

Eight studies reported sensitivity (mean 76\%, range $58-93 \%$ ), specificity (mean 48\%, range $24-69 \%$ ), PPV (mean 66\%, range 45-82\%), and NPV (mean 67\%, range 53-86\%) for this predictor. 


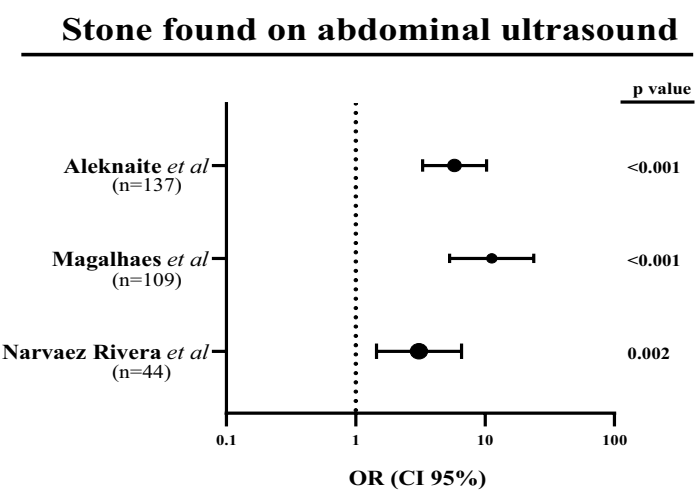

Total blirubin $>4 \mathrm{mg} / \mathrm{dL}$

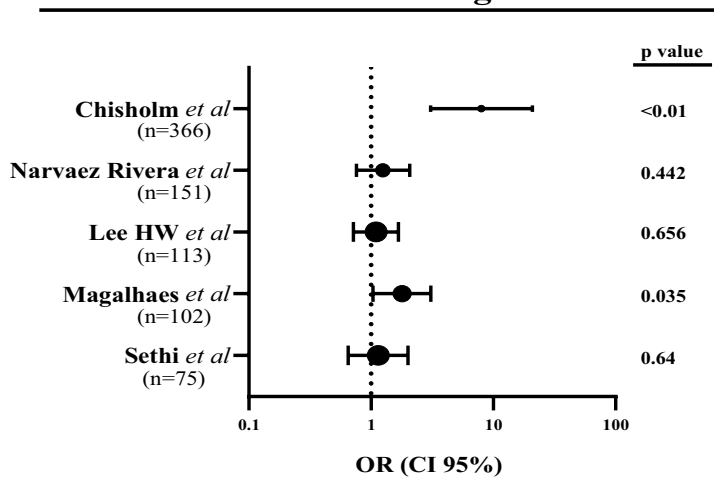

Common bile duct dilation $>6 \mathrm{~mm}$
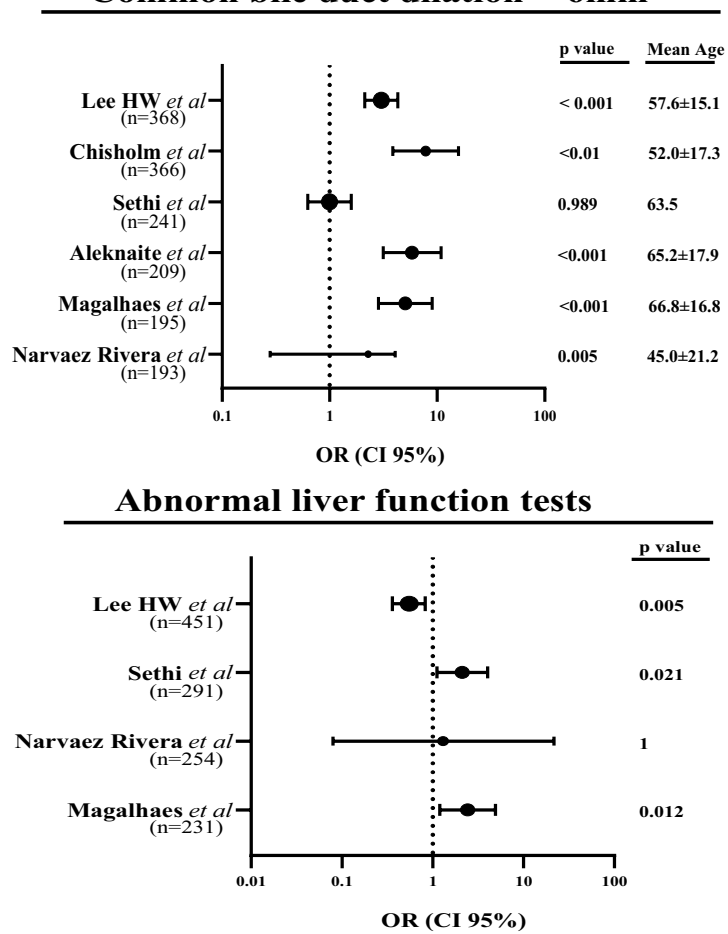

1 Cholangitis defined by diagnosis in medical record 2 Cholangitis defined by Charcot's triad

3 Cholangitis defined by upper abdominal pain, systemic infection, and abnormal liver function test markers

4 Cholangitis definition not reported

Fig. 3 Forest plots of studies with univariate analyzed OR reporting
Acute cholangitis

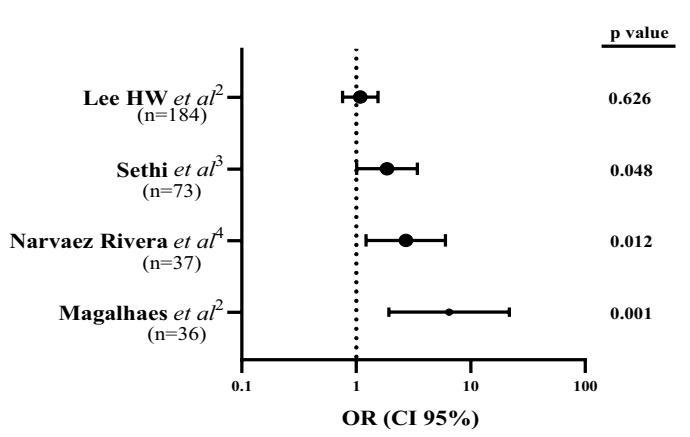

Total blirubin 1.8-4 mg/dL

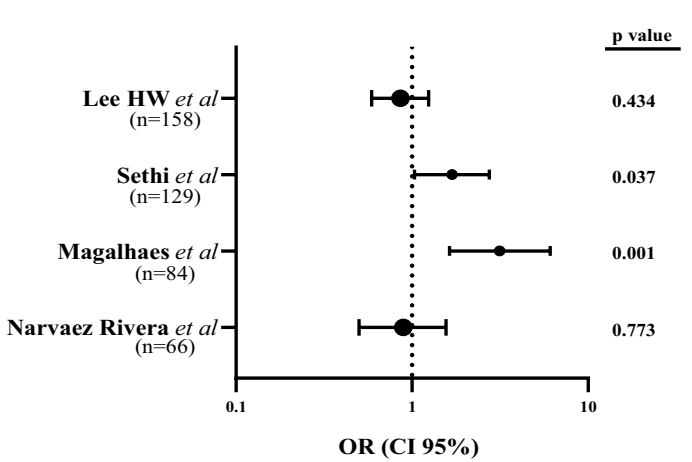

Age $>55$

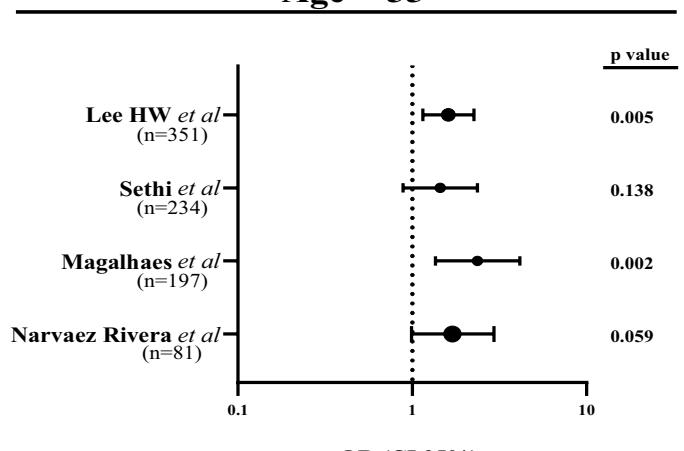

OR (CI 95\%)

Acute pancreatitis

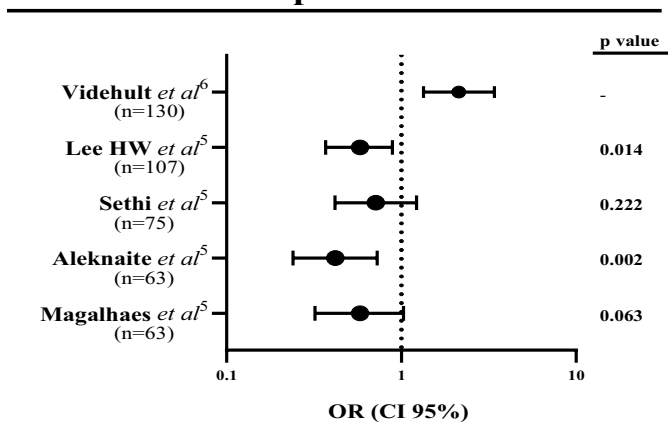

5 Pancreatitis defined by adnominal pain, imagine evidence, and lipase elevated three-fold

6 Pancreatitis defined by adnominal pain, imagine evidence, and lipase elevated four-fold 
Table 2 Detailed information of multivariate analyzed studies shown in Fig. 2

\begin{tabular}{|c|c|c|c|c|c|c|c|c|}
\hline Predictor & Study & $n$ & OR & CI lower $95 \%$ & CI upper $95 \%$ & $p$ value & Variance & Weight $(\%)$ \\
\hline \multirow[t]{5}{*}{ US with stone } & He et al. [21] & 524 & 17.3 & 12.6 & 23.8 & $<.0001$ & 13.8 & 7.25 \\
\hline & Kuzu et al. [22] & 285 & 2.74 & 1.63 & 4.60 & $<0.001$ & 19.9 & 5.01 \\
\hline & Panda et al. [23] & 152 & 42 & 4.56 & 386 & 0.001 & 195 & 0.51 \\
\hline & Rubin et al. [19] & 43 & 6.65 & 2.57 & 17.2 & $<0.001$ & 10.1 & 9.90 \\
\hline & Suarez et al. [24] & 13 & 6.4 & 1.5 & 27.3 & - & 7.12 & 14.04 \\
\hline \multirow[t]{5}{*}{ Acute cholangitis } & He et al. [21] & 463 & 0.9 & 0.6 & 1.1 & 0.25 & 11.1 & 9.03 \\
\hline & Kuzu et al. [22] & 154 & 1.2 & 0.59 & 2.42 & 0.617 & 19.8 & 5.04 \\
\hline & Jeon et al. [25] & 109 & 5.84 & 1.23 & 27.8 & 0.026 & 68.9 & 1.45 \\
\hline & Sethi et al. [26] & 73 & 1.50 & 0.77 & 2.93 & 0.23 & 8.46 & 11.82 \\
\hline & Rubin et al. [19] & 23 & 3.88 & 1.3 & 11.6 & 0.015 & 7.16 & 13.97 \\
\hline \multirow[t]{5}{*}{ Tbli $>4$ mg/dL } & He et al. [21] & 349 & 3.1 & 2.3 & 4.2 & $<.0001$ & 8.24 & 12.14 \\
\hline & Kuzu et al. [22] & 210 & 1.29 & 0.88 & 1.89 & 0.291 & 7.99 & 12.52 \\
\hline & Rubin et al. [19] & 167 & 2.67 & 1.8 & 3.97 & $<0.001$ & 6.80 & 14.71 \\
\hline & Sethi et al. [26] & 75 & 1.38 & 0.70 & 2.704 & 0.348 & 8.84 & 11.31 \\
\hline & Suarez et al. [24] & 37 & 4.85 & 1.82 & 12.9 & - & 9.25 & 10.81 \\
\hline \multirow[t]{3}{*}{ Tbli $1.8-4 \mathrm{mg} / \mathrm{dL}$} & Rubin et al. [19] & 172 & 0.88 & 0.61 & 1.27 & 0.483 & 6.02 & 16.61 \\
\hline & Kuzu et al. [22] & 164 & 1.48 & 0.89 & 2.45 & 0.131 & 10.9 & 9.13 \\
\hline & Sethi et al. [26] & 129 & 1.86 & 1.02 & 3.38 & 0.043 & 12.1 & 8.28 \\
\hline \multirow[t]{6}{*}{$\mathrm{CBD}>6 \mathrm{~mm}$} & He et al. [21] & 1419 & 1.80 & 1.50 & 2.20 & $<.0001$ & 13.5 & 7.38 \\
\hline & Kuzu et al. [22] & 780 & 2.54 & 1.55 & 4.16 & $<0.001$ & 49.5 & 2.02 \\
\hline & Rubin et al. [19] & 260 & 2.19 & 1.54 & 3.12 & $<0.001$ & 8.4 & 11.86 \\
\hline & Sethi et al. [26] & 241 & 0.97 & 0.58 & 1.61 & 0.89 & 16.3 & 6.14 \\
\hline & Jeon et al. [25] & 133 & 3.02 & 0.74 & 12.3 & 0.13 & 68.5 & 1.46 \\
\hline & Kamath et al. [27] & 77 & 1.95 & 1.13 & 20.2 & - & 41.6 & 2.41 \\
\hline \multirow[t]{4}{*}{ ALFT } & He et al. [21] & 1657 & 2.30 & 1.90 & 2.90 & $<.0001$ & 19.3 & 5.19 \\
\hline & Kuzu et al. [22] & 612 & 0.65 & 0.38 & 1.12 & 0.177 & 47.1 & 2.12 \\
\hline & Rubin et al. [19] & 499 & 2.88 & 1.15 & 7.18 & 0.023 & 108.9 & 0.92 \\
\hline & Sethi et al. [26] & 291 & 1.75 & 0.82 & 3.72 & 0.147 & 43.2 & 2.32 \\
\hline \multirow[t]{5}{*}{ Age $>55$} & He et al. [21] & 1408 & 1.40 & 1.20 & 1.70 & 0.0004 & 11.1 & 9.00 \\
\hline & Kuzu et al. [22] & 609 & 1.25 & 0.89 & 1.76 & 0.20 & 18.6 & 5.38 \\
\hline & Sethi et al. [26] & 234 & 1.26 & 0.74 & 2.16 & 0.39 & 17.4 & 5.76 \\
\hline & Jeon et al. [25] & 115 & 8.46 & 1.65 & 43.3 & 0.01 & 79.9 & 1.25 \\
\hline & Rubin et al. [19] & 86 & 1.38 & 0.86 & 2.23 & 0.181 & 5.08 & 19.7 \\
\hline \multirow[t]{4}{*}{ Acute pancreatitis } & He et al. [21] & 310 & 0.40 & 0.30 & 0.60 & $<.0001$ & 9.69 & 10.3 \\
\hline & Kuzu et al. [22] & 135 & 0.64 & 0.39 & 1.05 & 0.175 & 8.46 & 11.8 \\
\hline & Rubin et al. [19] & 134 & 0.63 & 0.42 & 0.94 & 0.022 & 5.66 & 17.7 \\
\hline & Sethi et al. [26] & 75 & 0.74 & 0.41 & 1.33 & 0.316 & 6.64 & 15.1 \\
\hline
\end{tabular}

\section{Abnormal liver function test (ALFT)}

Four studies reported multivariate analyzed ORs with a WaOR of 1.90. Sample sizes ranged from 291 [26] to 1657 [21]. Rubin et al. [19] (OR 2.88; [95\% CI 1.15-7.18]) reported the highest OR, while Kuzu et al. [22] (OR 0.65; [95\% CI 0.38-1.12]) reported the lowest OR. He et al. [21] (OR 2.3; [95\% CI 1.9-2.9]) carried the most inverse-variance weight $(5.2 \%)$.

Four studies reported univariate analyzed ORs with a WaOR of 1.54. Sample sizes ranged from 231[18] to 451
[29]. Magalhaes et al. [18] (OR 2.43; [95\% CI 1.2-4.9]) reported the highest OR, while Lee et al. [29] (OR 0.55; [95\% CI 0.36-0.83]) reported the lowest OR and carried the most inverse-variance weight (4.9\%) among these three studies.

Seven studies reported sensitivity (mean $85 \%$, range $71-99 \%$ ), specificity (mean $25 \%$, range $1-59 \%$ ), PPV (mean 58\%, range 35-82\%), and NPV (mean 56\%, range 26-92\%) for this predictor. 
Table 3 Detailed information of univariate analyzed studies shown in Fig. 3

\begin{tabular}{|c|c|c|c|c|c|c|c|c|}
\hline Predictor & Study & $n$ & OR & CI lower $95 \%$ & CI upper $95 \%$ & $p$ value & Variance & Weight $(\%)$ \\
\hline \multirow[t]{3}{*}{ US with stone } & Aleknaite et al. [28] & 137 & 5.81 & 3.3 & 10.3 & $<0.001$ & 11.5 & 8.72 \\
\hline & Magalhaes et al. [18] & 109 & 11.3 & 5.32 & 23.8 & $<0.001$ & 15.9 & 6.28 \\
\hline & Nárvaez Rivera et al. [14] & 44 & 3.09 & 1.45 & 6.58 & 0.002 & 6.55 & 15.27 \\
\hline \multirow[t]{4}{*}{ Acute cholangitis } & Lee et al. [29] & 184 & 1.09 & 0.76 & 1.55 & 0.626 & 6.08 & 16.44 \\
\hline & Magalhaes et al. [18] & 36 & 6.48 & 1.93 & 21.8 & 0.001 & 13.8 & 7.26 \\
\hline & Nárvaez Rivera et al. [14] & 37 & 2.71 & 1.22 & 6.01 & 0.012 & 6.12 & 16.33 \\
\hline & Sethi et al. [26] & 73 & 1.85 & 1.01 & 3.412 & 0.048 & 7.07 & 14.14 \\
\hline \multirow[t]{5}{*}{ Tbli > 4 mg/dL } & Chisholm et al. [30] & 366 & 7.97 & 3.06 & 20.8 & $<0.01$ & 87.5 & 1.14 \\
\hline & Nárvaez Rivera et al. [14] & 151 & 1.25 & 0.76 & 2.07 & 0.442 & 9.87 & 10.14 \\
\hline & Lee et al. [29] & 113 & 1.1 & 0.72 & 1.67 & 0.656 & 5.21 & 19.21 \\
\hline & Magalhaes et al. [18] & 102 & 1.79 & 1.04 & 3.08 & 0.035 & 7.85 & 12.78 \\
\hline & Sethi et al. [26] & 75 & 1.14 & 0.65 & 2.00 & 0.64 & 6.13 & 16.31 \\
\hline \multirow[t]{4}{*}{ Tbli $1.8-4$ mg/dL } & Lee et al. [29] & 158 & 0.86 & 0.59 & 1.24 & 0.434 & 5.67 & 17.63 \\
\hline & Sethi et al. [26] & 129 & 1.69 & 1.03 & 2.75 & 0.037 & 8.07 & 12.39 \\
\hline & Magalhaes et al. [18] & 84 & 3.15 & 1.63 & 6.08 & 0.001 & 9.47 & 10.56 \\
\hline & Nárvaez Rivera et al. [14] & 66 & 0.89 & 0.5 & 1.56 & 0.773 & 5.56 & 17.98 \\
\hline \multirow[t]{6}{*}{$\mathrm{CBD}>6 \mathrm{~mm}$} & Lee et al. [29] & 368 & 3.03 & 2.12 & 4.30 & $<0.001$ & 12.0 & 8.35 \\
\hline & Chisholm et al. [30] & 366 & 7.83 & 3.87 & 15.8 & $<0.01$ & 47.3 & 2.11 \\
\hline & Sethi et al. [26] & 241 & 1.00 & 0.63 & 1.59 & 0.99 & 13.5 & 7.41 \\
\hline & Aleknaite et al. [28] & 209 & 5.85 & 3.15 & 10.9 & $<0.001$ & 21.0 & 4.77 \\
\hline & Magalhaes et al. [18] & 195 & 5.06 & 2.85 & 8.99 & $<0.001$ & 16.7 & 5.97 \\
\hline & Nárvaez Rivera et al. [14] & 193 & 2.28 & 0.28 & 4.08 & 0.01 & 90.1 & 1.11 \\
\hline \multirow[t]{4}{*}{ ALFT } & Lee et al. [29] & 451 & 0.55 & 0.36 & 0.83 & 0.005 & 20.5 & 4.88 \\
\hline & Sethi et al. [26] & 291 & 2.12 & 1.12 & 4.01 & 0.021 & 30.8 & 3.36 \\
\hline & Nárvaez Rivera et al. [14] & 254 & 1.30 & 0.08 & 21.60 & 1 & 518.1 & 0.19 \\
\hline & Magalhaes et al. [18] & 231 & 2.43 & 1.20 & 4.90 & 0.01 & 29.8 & 3.25 \\
\hline \multirow[t]{4}{*}{ Age $>55$} & Lee et al. [29] & 351 & 1.61 & 1.15 & 2.26 & 0.005 & 10.4 & 9.59 \\
\hline & Sethi et al. [26] & 234 & 1.45 & 0.89 & 2.37 & 0.138 & 14.7 & 6.81 \\
\hline & Magalhaes et al. [18] & 197 & 2.37 & 1.36 & 4.15 & 0.002 & 16.0 & 6.27 \\
\hline & Nárvaez Rivera et al. [14] & 81 & 1.70 & 0.99 & 2.94 & 0.059 & 6.25 & 16.0 \\
\hline \multirow[t]{5}{*}{ Acute pancreatitis } & Videhult et al. [17] & 130 & 2.13 & 1.34 & 3.41 & - & 7.38 & 13.6 \\
\hline & Lee et al. [29] & 107 & 0.58 & 0.37 & 0.89 & 0.014 & 5.36 & 18.6 \\
\hline & Sethi et al. [26] & 75 & 0.72 & 0.42 & 1.23 & 0.222 & 5.64 & 17.7 \\
\hline & Aleknaite et al. [28] & 63 & 0.42 & 0.24 & 0.73 & 0.002 & 5.07 & 19.7 \\
\hline & Magalhaes et al. [18] & 63 & 0.58 & 0.32 & 1.03 & 0.063 & 5.60 & 17.9 \\
\hline
\end{tabular}

\section{Age $>55$}

Five studies reported multivariate analyzed ORs with a WaOR of 1.57. Sample sizes ranged from 86 [19] to 1408 [21]. Jeon et al. [25] (OR 8.46; [95\% CI 1.65- 43.3]) reported the highest OR, while Kuzu et al. [22] (OR 1.25; [95\% CI 0.89-1.76]) reported the lowest OR. Rubin et al. [19] (OR 1.38; [95\% CI 0.86-2.23]) carried the most inverse variance weight (20\%).

Four studies reported univariate analyzed ORs with a WaOR of 1.74. Sample sizes ranged from 81 [14] to 351 [29]. Magalhaes et al. [18] (OR 2.37; [95\% CI 1.36-4.15]) reported highest OR, while Sethi et al. (OR 1.45; [95\% CI
0.89-2.37]) reported the lowest OR. Nárvaez Rivera et al. [14] (OR 1.7; [95\% CI 0.99-2.94]) carried the most inversevariance weight (16\%) among these three studies.

Seven studies reported sensitivity (mean 53\%, range $18-79 \%$ ), specificity (mean 59\%, range 35-86\%), PPV (mean $60 \%$, range $30-80 \%$ ), and NPV (mean $51 \%$, range $23-75 \%)$ for this predictor.

\section{Acute pancreatitis}

Four studies reported multivariate analyzed ORs with a WaOR of 0.62. Sample sizes ranged from 75 [26] to 310 [21]. Sethi et al. [26] (OR 0.74; [95\% CI 0.41-1.33]) 
Table 4 Studies reported sensitivity, specificity, PPV, and NPV for each ASGE predictor

\begin{tabular}{|c|c|c|c|c|c|c|}
\hline Predictor & Study & $n$ & Sensitivity (\%) & Specificity (\%) & $\begin{array}{l}\text { Positive predictive } \\
\text { value }(\%)\end{array}$ & $\begin{array}{l}\text { Negative pre- } \\
\text { dictive value } \\
(\%)\end{array}$ \\
\hline \multirow[t]{11}{*}{ US with stone } & Yang et al. [31] & 926 & 35.7 & 97.9 & 58.1 & 94.9 \\
\hline & He et al. [21] & 524 & 44 & 97 & 91 & 73 \\
\hline & Kuzu et al. [22] & 285 & 36.6 & 85.3 & 90.5 & 26 \\
\hline & Jagtap et al. [16] & 174 & 62 & 99.6 & 98.3 & 87.9 \\
\hline & Aleknaite et al. [28] & 137 & 51.3 & 84.6 & 86.2 & 48.3 \\
\hline & Magalhaes et al. [18] & 109 & 55.9 & 89.9 & 91.7 & 50.3 \\
\hline & Bose et al. [32] & 88 & 50 & 97 & 87 & 82 \\
\hline & Adams et al. [13] & 65 & 21.8 & 93.5 & 70.8 & 62.3 \\
\hline & Nárvaez Rivera et al. [14] & 44 & 23 & 91 & 77 & 48 \\
\hline & Rubin et al. [19] & 43 & 13 & 98 & 88 & 47 \\
\hline & Suarez et al. [24] & 13 & 14.1 & 97.1 & 76.9 & 61.9 \\
\hline \multirow[t]{9}{*}{ Acute cholangitis } & He et al. [21] & 463 & 20 & 84 & 44 & 61 \\
\hline & Videhult et al. [17] & 323 & 22 & 70 & 9 & 88 \\
\hline & Lee et al. [29] & 184 & 32.4 & 69.6 & 59.8 & 42.4 \\
\hline & Kuzu et al. [22] & 154 & 18.9 & 88.6 & 86.3 & 22.1 \\
\hline & Bose et al. [32] & 88 & 42 & 100 & 100 & 80 \\
\hline & Jagtap et al. [16] & 71 & 22.8 & 99 & 88.7 & 78.1 \\
\hline & Nárvaez Rivera et al. [14] & 37 & 19 & 92 & 76 & 47 \\
\hline & Magalhaes et al. [18] & 36 & 18.4 & 96.6 & 91.7 & 37 \\
\hline & Rubin et al. [19] & 23 & 7 & 98 & 83 & 45 \\
\hline \multirow[t]{8}{*}{ Tbli > 4 mg/dL } & He et al. [21] & 349 & 22 & 94 & 69 & 65 \\
\hline & Kuzu et al. [22] & 210 & 25.2 & 82.6 & 84.7 & 22.4 \\
\hline & Rubin et al. [19] & 167 & 41 & 79 & 72 & 51 \\
\hline & Nárvaez Rivera et al. [14] & 151 & 61 & 44 & 59 & 47 \\
\hline & Lee et al. [29] & 113 & 20 & 81.5 & 60.2 & 42.1 \\
\hline & Magalhaes et al. [18] & 102 & 42.5 & 70.8 & 74.5 & 37.8 \\
\hline & Suarez et al. [24] & 37 & 29.6 & 84.3 & 56.8 & 63.2 \\
\hline & Chan et al. [33] & 19 & 39 & 95 & 93 & 44 \\
\hline \multirow[t]{5}{*}{ Tbli $1.8-4$ mg/dL } & Rubin et al. [19] & 172 & 32 & 63 & 54 & 43 \\
\hline & Kuzu et al. [22] & 164 & 19.5 & 85.3 & 83.5 & 21.6 \\
\hline & Lee et al. [29] & 158 & 25.9 & 71.2 & 55.7 & 40.71 \\
\hline & Magalhaes et al. [18] & 84 & 61.1 & 66.6 & 75 & 51.2 \\
\hline & Nárvaez Rivera et al. [14] & 66 & 25 & 73 & 55 & 53 \\
\hline \multirow[t]{8}{*}{$\mathrm{CBD}>6 \mathrm{~mm}$} & He et al. [21] & 1419 & 75 & 63 & 57 & 79 \\
\hline & Kuzu et al. [22] & 780 & 70.9 & 23.9 & 82 & 70.4 \\
\hline & Jagtap et al. [16] & 434 & 69.9 & 68.5 & 44.5 & 86.4 \\
\hline & Lee et al. [29] & 368 & 73.8 & 51.9 & 68.2 & 58.6 \\
\hline & Rubin et al. [19] & 260 & 58 & 61 & 66 & 53 \\
\hline & Aleknaite et al. [28] & 209 & 92.5 & 32.2 & 71.3 & 70.2 \\
\hline & Magalhaes et al. [18] & 195 & 83.8 & 49.4 & 76.9 & 60.3 \\
\hline & Nárvaez Rivera et al. [14] & 193 & 82 & 33 & 62 & 59 \\
\hline \multirow[t]{7}{*}{ ALFT } & He et al. [21] & 1657 & 77 & 50 & 50 & 77 \\
\hline & Kuzu et al. [22] & 612 & 71.1 & 39.6 & 81.8 & 26.4 \\
\hline & Rubin et al. [19] & 499 & 98 & 7 & 57 & 68 \\
\hline & Lee et al. [29] & 451 & 73.2 & 16.9 & 55.2 & 31.1 \\
\hline & Nárvaez Rivera et al. [14] & 254 & 99 & 1 & 57 & 50 \\
\hline & Magalhaes et al. [18] & 231 & 89.9 & 21.3 & 69.7 & 51.3 \\
\hline & Jagtap et al. [16] & 205 & 90.2 & 38.5 & 34.6 & 91.6 \\
\hline
\end{tabular}


Table 4 (continued)

\begin{tabular}{|c|c|c|c|c|c|c|}
\hline Predictor & Study & $n$ & Sensitivity (\%) & Specificity (\%) & $\begin{array}{l}\text { Positive predictive } \\
\text { value }(\%)\end{array}$ & $\begin{array}{l}\text { Negative pre- } \\
\text { dictive value } \\
(\%)\end{array}$ \\
\hline \multirow[t]{7}{*}{ Age $>55$} & He et al. [21] & 1408 & 60 & 54 & 46 & 67 \\
\hline & Kuzu et al. [22] & 609 & 69.6 & 35.3 & 80.4 & 23.3 \\
\hline & Lee et al. [29] & 351 & 65 & 46.5 & 62.3 & 48.7 \\
\hline & Jagtap et al. [16] & 205 & 37 & 69.5 & 30.4 & 75.4 \\
\hline & Magalhaes et al. [18] & 197 & 79.3 & 38.2 & 72.1 & 47.9 \\
\hline & Rubin et al. [19] & 86 & 18 & 86 & 63 & 45 \\
\hline & Nárvaez Rivera et al. [14] & 81 & 37 & 85 & 65 & 47 \\
\hline \multirow[t]{11}{*}{ Acute pancreatitis } & Jagtap et al. [16] & 408 & 23.2 & 55.9 & 15.7 & 66.6 \\
\hline & He et al. [21] & 310 & 10 & 85 & 29 & 59 \\
\hline & Kuzu et al. [22] & 135 & 13.6 & 78.8 & 71.1 & 19.3 \\
\hline & Rubin et al. [19] & 134 & 22 & 69 & 48 & 41 \\
\hline & Videhult et al. [17] & 130 & 21 & 90 & 20 & 90 \\
\hline & Lee et al. [29] & 107 & 15 & 77 & 47.7 & 39.3 \\
\hline & Bose et al. [32] & 88 & 12 & 96 & 60 & 72 \\
\hline & Nárvaez Rivera et al. [14] & 80 & 20 & 54 & 36 & 34 \\
\hline & Magalhaes et al. [18] & 63 & 20.1 & 69.7 & 57.1 & 30.2 \\
\hline & Adams et al. [13] & 57 & 41.7 & 68.9 & 43.9 & 67 \\
\hline & Suarez et al. [24] & 56 & 55.6 & 76.3 & 52.6 & 78.4 \\
\hline $\begin{array}{l}\text { Tbli }>4 \mathrm{mg} / \mathrm{dL}+ \\
\mathrm{CBD}>6 \mathrm{~mm}\end{array}$ & He et al. [21] & 267 & 19 & 96 & 78 & 58 \\
\hline
\end{tabular}

Sample sizes reported were relevant sample sizes, as not all studies had samples comprised entirely of (suspected) CDL patients

reported the highest OR, while $\mathrm{He}$ et al. [21] (OR 0.4; [95\% CI 0.3-0.6]) reported the lowest OR. Rubin et al. [19] (OR 0.63; [95\% CI 0.42-0.94]) carried the most inverse variance weight (18\%).

Five studies reported univariate analyzed ORs with a WaOR of 0.811 . Sample sizes ranged from 63 [18, 28] to 130 [17]. Videhult et al. [17] (OR 2.13; [95\% CI 1.34-3.41]) reported the highest OR, while Aleknaite et al. [28] (OR 0.42; [95\% CI 0.24-0.73]) reported the lowest OR. Lee et al. [29] (OR 0.58; [95\% CI 0.37-0.89]) carried the most inverse-variance weight (19\%) among these three studies.

Eleven studies reported sensitivity (mean 23\%, range $10-56 \%$ ), specificity (mean $75 \%$, range $54-96 \%$ ), PPV (mean 44\%, range 16-71\%), and NPV (mean 54\%, range $19-90 \%)$ for this predictor.

\section{Total bilirubin $>4 \mathrm{mg} / \mathrm{dL}$ and $\mathrm{CBD}>6 \mathrm{~mm}$}

He et al. [21] was the only study that reported diagnostic performance of this newly proposed predictor from the 2019 ASGE guideline. The study reported a sample size of 267 , sensitivity $19 \%$, specificity $96 \%$, PPV $78 \%$, and NPV 58\%.

\section{Discussion}

In this systematic review, we aimed to identify studies that examined the predictive performance of the 2010 ASGE guideline CDL predictors and helped contributing to the changes proposed on the 2019 ASGE guideline. As the result, we excluded all publications that did not report 'predict' and 'associate' in their title or abstract. Our key findings include: (i) "US with stone" demonstrated outstanding predictive value compared to other predictors, (ii) $\mathrm{CBD}>6$ and $\mathrm{AC}$ demonstrated similar predictive value for CDL, potentially calling into question the modification made in the 2019 ASGE guideline, (iii) Tbili is not reliable for CDL when used alone, and (iv) AP demonstrated a negative predictive trend for CDL.

A high-level summary of the findings of this study is provided on Table 6. With a high WaOR in both univariate and multivariate analyses (Table 1), our review showed that "US with stone" had a significantly higher predictive value for CDL than all other predictors, including their peer predictors Tbili $>4$ and AC. With a low reported sensitivity, this predictor is less helpful in ruling out CDL; however, a high reported specificity provides strength for ruling in CDL. The forest plots (Figs. 1,2) and $p$ values 
Table 5 Characteristics of included studies

\begin{tabular}{|c|c|c|c|c|c|c|}
\hline Study & Publication year & Journal & Study type & $\begin{array}{l}\text { Total } \\
\text { sample } \\
\text { size }\end{array}$ & Study demographic & Gold standard \\
\hline Adams et al. [13] & 2015 & $\begin{array}{l}\text { Gastrointestinal Endos- } \\
\text { copy }\end{array}$ & Retrospective cohort & 498 & USA & ERCP \\
\hline Aleknaite et al. [28] & 2018 & $\begin{array}{l}\text { United European Gastro- } \\
\text { enterology Journal }\end{array}$ & Retrospective cohort & 350 & Lithuania & ERCP \& IOC \\
\hline Bose et al. [32] & 2001 & Surgery Today & Prospective cohort & 88 & India & ERCP \& IOC \\
\hline Chan et al. [33] & 2008 & $\begin{array}{l}\text { The American Journal of } \\
\text { Surgery }\end{array}$ & Retrospective cohort & 182 & USA & ERCP \\
\hline Chisholm et al. [30] & 2019 & $\begin{array}{l}\text { Gastrointestinal Endos- } \\
\text { copy }\end{array}$ & Prospective cohort & 366 & USA & ERCP \\
\hline He et al. [21] & 2017 & $\begin{array}{l}\text { Gastrointestinal Endos- } \\
\text { copy }\end{array}$ & Prospective cohort & 2724 & China & ERCP \\
\hline Jagtap et al. [16] & 2020 & Endoscopy & Prospective cohort & 1042 & India & ERCP \\
\hline Jeon et al. [25] & 2017 & Gut and Liver & Retrospective cohort & 200 & Korea & ERCP \\
\hline Kamath et al. [27] & 2016 & $\begin{array}{l}\text { Indian Journal of Gastro- } \\
\text { enterology }\end{array}$ & Prospective cohort & 275 & Saudi Arabia & ERCP \\
\hline Kuzu et al. [22] & 2017 & НPB & Retrospective cohort & 1074 & Turkey & ERCP \\
\hline Lee et al. [29] & 2019 & $\begin{array}{l}\text { Hepatobiliary \& Pancre- } \\
\text { atic Diseases Interna- } \\
\text { tional }\end{array}$ & Retrospective cohort & 754 & Korea & ERCP \\
\hline Magalhaes et al. [18] & 2015 & $\begin{array}{l}\text { World Journal of Gastro- } \\
\text { intestinal Endoscopy }\end{array}$ & Retrospective cohort & 268 & Portugal & ERCP \\
\hline Nárvaez Rivera et al. [14] & 2016 & $\begin{array}{l}\text { Spanish Journal of Gas- } \\
\text { troenterology }\end{array}$ & Prospective cohort & 256 & Spain & ERCP \\
\hline Panda et al. [23] & 2018 & World Journal of Surgery & Retrospective cohort & 152 & USA & ERCP \\
\hline Rahal et al. [34] & 2017 & $\begin{array}{l}\text { European Journal of } \\
\text { Gastroenterology \& } \\
\text { Hepatology }\end{array}$ & Retrospective cohort & 354 & Lebanon & ERCP \& IOC \\
\hline Rubin et al. [19] & 2013 & Digestive Endoscopy & Retrospective cohort & 1080 & USA & ERCP \\
\hline Sethi et al. [26] & 2016 & Digestive Endoscopy & Prospective cohort & 336 & USA & ERCP \\
\hline Suarez et al. [24] & 2016 & Surgical Endoscopy & Prospective cohort & 173 & USA & ERCP \\
\hline Videhult et al. [17] & 2011 & HРB & Prospective cohort & 1171 & Sweden & IOC \\
\hline Yang et al. [31] & 2008 & Surgical Endoscopy & Retrospective cohort & 2225 & USA & Cholecystectomy \\
\hline
\end{tabular}

Sample sizes reported were total sample sizes, including patients not entirely of (suspected) CDL patients. Specific relevant sample size is reported in Table 2, 3, 4 for each specific predictor

further support this observation. "US with stone" has also been reported to have high specificity (94\%) and PPV (81\%), significant for ruling in CDL. Spontaneous passage of stone before ERCP may explain why PPV, though high, is typically not $100 \%$. Although $\mathrm{AC}$ and Tbili $>4 \mathrm{mg} /$ dL did not perform as strongly as "US with stone," they demonstrated a relatively higher predictive value than all other predictors. However, Tbili $>4 \mathrm{mg} / \mathrm{dL}$ had a lower predictive value, demonstrated by its $\mathrm{WaOR}$ in univariate analysis, when used alone. This finding is not unexpected, as multiple factors and pre-existing conditions can lead to elevated Tbili levels.

Despite being in the same group of "strong predictor," $\mathrm{CBD}>6 \mathrm{~mm}$ demonstrated a stronger predictive value than Tbili 1.8-4 mg/dL (Table 1). It's worth noting that
CBD $>6 \mathrm{~mm}$ might be more useful in a younger patient population, as CBD diameter can gradually increase with age. We also found that CBD $>6 \mathrm{~mm}$ had high sensitivity (76\%) and similar PPV (66\%) and NPV (67\%). This finding potentially indicates that the absence of CBD dilation is helpful in ruling out CDL. Based on the WaOR, Tbili $1.8-4 \mathrm{mg} / \mathrm{dL}$ demonstrated less predictive value than its peer and performed worse than the "moderate predictors," ALFT and age $>55$. This finding potentially invalidates the role of Tbili 1.8-4 as a strong predictor; however, our review found fewer studies that reported data for Tbili $1.8-4 \mathrm{mg} / \mathrm{dL}$, which limits interpretation. Moreover, relatively high specificity (72\%) was observed with Tbili $1.8-4 \mathrm{mg} / \mathrm{dL}$, potentially indicating some predictive value. 
Table 6 Comparison of various CDL predictors between the 2010 and the 2019 ASGE guidelines with study resulted recommendation and comments

\begin{tabular}{|c|c|c|c|}
\hline Predictors & $\begin{array}{l}2010 \text { ASGE guide- } \\
\text { line CDL predictor } \\
\text { category }\end{array}$ & $\begin{array}{l}2019 \text { ASGE guide- } \\
\text { line CDL predictor } \\
\text { category }\end{array}$ & Comment based on study result \\
\hline US with stone & Very strong & High & Highest WaOR of all predictors \\
\hline Acute cholangitis & Very strong & High & $\begin{array}{l}\text { Lower WaOR than US with stone; however, still relatively higher } \mathrm{WaOR} \\
\text { than other predictors }\end{array}$ \\
\hline Tbili > $4 \mathrm{mg} / \mathrm{dL}$ & Very strong & Not reported & Equivalent multivariate analysis $\mathrm{WaOR}$ as compared to acute cholangitis \\
\hline $\begin{array}{l}\text { Tbili }>4 \mathrm{mg} / \mathrm{dL} \\
+\mathrm{CBD}>6 \mathrm{~mm}\end{array}$ & Not reported & High & Insufficient data to comment; suspect higher specificity than either alone \\
\hline Tbili $1.8-4 \mathrm{mg} / \mathrm{dL}$ & Strong & Not reported & $\begin{array}{l}\text { Relatively low WaOR, equivalent to moderate predictors reported in } 2010 \\
\text { ASGE guideline. However, limited studies }\end{array}$ \\
\hline $\mathrm{CBD}>6 \mathrm{~mm}$ & Strong & Intermediate & $\begin{array}{l}\text { High WaOR on univariate analysis, low WaOR on multivariate analysis. } \\
\text { Relatively high sensitivity and NPV }\end{array}$ \\
\hline ALFT & Moderate & Intermediate & Low WaOR, nonspecific \\
\hline Age $>55$ years & Moderate & Intermediate & Low WaOR, nonspecific \\
\hline Acute pancreatitis & Moderate & Not reported & $\mathrm{WaOR}<1$, indicates a negative predictor of $\mathrm{CDL}$ \\
\hline
\end{tabular}

It is presumed that the term "likelihood" reported in the 2010 ASGE guideline and "probability" reported in the 2019 ASGE guideline have analogous meanings and, similarly, that the strength qualifiers (e.g. "very strong") reported in the 2010 ASGE guideline and probability (e.g. "high") qualifiers reported in the 2019 ASGE guideline have analogous meanings

ALFT is loosely defined. It can refer to any abnormal liver biochemical value other than bilirubin. Our review demonstrated that ALFT has a similar predictive value as $\mathrm{CBD}>6 \mathrm{~mm}$ in multivariate analysis and has relatively higher sensitivity, potentially indicating some added clinical value. However, when used alone, the predictive value was relatively low. Overall, the predictive value of ALFT is difficult to determine given its imprecise definition. Additional studies are needed to investigate the role of different biochemical markers and their temporal trend in CDL prediction.

Age $>55$ demonstrated minimal predictive value with its relatively weak WaOR, similar to ALFT. Furthermore, Table 4 shows no standout with any of the four other statistical factors, further limiting its predictive value. With regard to $\mathrm{AP}$, our review revealed a $\mathrm{WaOR}<1$, indicating a negative association, suggested that (persistence of) CDL is perhaps less likely in patients with AP. Concordantly, the 2019 ASGE guideline no longer includes AP as a CDL predictor or risk criterion.

In summary, the 2019 ASGE guideline designates $\mathrm{AC}$ and "US with stone" as a high-risk predictors and no longer has Tbili $>4 \mathrm{mg} / \mathrm{dL}$ alone as a "strong" predictor, instead requiring it to be in combination with CBD dilation to be considered a composite "high-risk" predictor. The 2019 guideline also downgrades CBD dilation alone to the "intermediate-risk" group and removes AP and Tbili $1.8-4 \mathrm{mg} / \mathrm{dL}$ altogether. Our study lacked predictive data for the grouped predictor "CBD dilation with Tbili $>4 \mathrm{mg} /$ dL" except for one study [21]. The recommendation in the presence of a "high-risk" predictor is to proceed to ERCP, while additional imaging modalities are recommended for "intermediate-risk" predictors. Overall, our review supports the conclusions drawn by many of the subject studies that the 2010 ASGE guideline CDL predictors had gaps in accuracy and evidence base.

This systematic review has limitations. Common with systematic reviews, missing data continues to be a challenge with the extraction process. To make efforts in retrieve any missing data and avoid attrition bias, the finalized data extraction results were crossed checked by reviewers (SM and LW). Despite including studies from a variety of geographic and demographic populations, some populations were better represented than others. Our study attempted to perform meta-analysis on a heterogeneous population, given the variability in clinical courses and data reporting. This heterogeneity creates subjective bias potentially confounding the scandalization process. Additionally, we excluded all non-English studies. These factors may decrease the generalizability of our findings. Additionally, most of the studies reported were from the last decade and the USA, which may further limit generalizability. Some predictors, for example Tbili $1.8-4 \mathrm{mg} / \mathrm{dL}$, had a relative paucity of data to analyze, thus limiting interpretation. Furthermore, as the review heavily involved literature search and data extraction, this process could generate human error. In the risk of bias assessment, we recognize that our study is subject to selective outcome reporting, as our exclusion criteria might omit to report related to our scope of the investigation, attrition bias, as limited by human error in the data extraction process. Lastly, our study is prone to detection biases and publication error associated with forest plots, 
as the weight of each standardized OR is dependent to its relative sample size, which is subject to further confounding and biases within the reporting studies. Our secondary outcomes, including sensitivity, specificity, PPV, and NPV, lacked a standardized statistical analysis due to limitations of the available data in the included studies.

Our study reviewed the predictive value of individual non-invasive CDL predictors. However, in clinical practice, CDL diagnosis is often made with a combination of factors. A liver function panel reports multiple predictors, including Tbili and liver enzymes. A right-upper-quadrant abdominal ultrasound not only assesses for stone presence but also attempts to visualize the degree of CBD dilation. As our findings indicated, some predictors excel in ruling out CDL with high sensitivity and NPV, while others excel in ruling in CDL with high specificity and PPV. Thus, it is important clinicians rely on more than one predictor in managing patients with suspicion of CDL. Though further study remains needed and dynamic changes in predictors should be considered on a case-by-case clinical basis, until such data become available, our review largely supports the modifications made to CDL predictors in the 2019 ASGE guideline as evidence-based.

Acknowledgements We thank UCLA Clinical and Translational Science Institute for providing the biostatistical support through the NIH National Center for Advancing Translational Science (NCATS). UCLA CTSI Grant Number UL1TR001881.

Author contributions Drafting of manuscript text: LW. Critical revision for intellectual content: TD, FC, JHT. Analysis for statistical methods: HW. Manuscript proofreading: SM, MG, CC. Final Approval: FC, ITM, HMC, AES, JHT.

\section{Declarations}

Conflict of interest No disclosures or conflicts of interest relevant to this submission. An abstract of this study was presented at DDW 2021 as an ASGE poster.

Ethical approval This submitting study is a retrospective systematic review of a series of findings from published primary articles. This study does not involve any human participants, patient data, or biological materials. This study did not meet the criteria that required ethnical committee from the primary academic institute David Geffen School of Medicine at UCLA.

Informed consent Informed consent was obtained from all individual participants included in the study.

Open Access This article is licensed under a Creative Commons Attribution 4.0 International License, which permits use, sharing, adaptation, distribution and reproduction in any medium or format, as long as you give appropriate credit to the original author(s) and the source, provide a link to the Creative Commons licence, and indicate if changes were made. The images or other third party material in this article are included in the article's Creative Commons licence, unless indicated otherwise in a credit line to the material. If material is not included in the article's Creative Commons licence and your intended use is not permitted by statutory regulation or exceeds the permitted use, you will need to obtain permission directly from the copyright holder. To view a copy of this licence, visit http://creativecommons.org/licenses/by/4.0/.

\section{References}

1. Everhart JE, Khare M, Hill M, et al. Prevalence and ethnic differences in gallbladder disease in the United States. Gastroenterology. 1999;117:632-9.

2. Schirmer BD, Winters KL, Edlich RF. Cholelithiasis and cholecystitis. J Long Term Eff Med Implants. 2005;15:329-38.

3. Peery AF, Crockett SD, Murphy CC, et al. Burden and cost of gastrointestinal, liver, and pancreatic diseases in the United States: update 2018. Gastroenterology. 2019;156:254-272.e11.

4. Frossard JL, Hadengue A, Amouyal G, et al. Choledocholithiasis: a prospective study of spontaneous common bile duct stone migration. Gastrointest Endosc. 2000;51:175-9.

5. Tranter SE, Thompson MH. Spontaneous passage of bile duct stones: frequency of occurrence and relation to clinical presentation. Ann R Coll Surg Engl. 2003;85:174-7.

6. Yadav D, Agarwal N, Pitchumoni CS. A critical evaluation of laboratory tests in acute pancreatitis. Am J Gastroenterol. 2002;97:1309-18.

7. Millat B, Borie F, Decker G. Treatment of choledocholithiasis: therapeutic ERCP versus peroperative extraction during laparoscopic cholecystectomy. Acta Gastroenterol Belg. 2000;63:301-3.

8. Lynn AP, Chong G, Thomson A. Endoscopic retrograde cholangiopancreatography in the treatment of intraoperatively demonstrated choledocholithiasis. Ann R Coll Surg Engl. 2014;96:45-8.

9. Jones WB, Blackwell J, McKinley B, et al. What is the risk of diagnostic endoscopic retrograde cholangiopancreatography before cholecystectomy? Am Surg. 2014;80:746-51.

10. Plewka M, Rysz J, Kujawski K. Complications of endoscopic retrograde cholangiopancreatography. Pol Merkur Lekarski. 2017;43:272-5.

11. Andriulli A, Loperfido S, Napolitano G, et al. Incidence rates of post-ERCP complications: a systematic survey of prospective studies. 2007. In: Database of Abstracts of Reviews of Effects (DARE): Quality-assessed Reviews [Internet]. York (UK): Centre for Reviews and Dissemination (UK); 1995.

12. ASGE Standards of Practice Committee, Maple JT, et al. The role of endoscopy in the evaluation of suspected choledocholithiasis. Gastrointest Endosc. 2010;71:1-9.

13. Adams MA, Hosmer AE, Wamsteker EJ, et al. Predicting the likelihood of a persistent bile duct stone in patients with suspected choledocholithiasis: accuracy of existing guidelines and the impact of laboratory trends. Gastrointest Endosc. 2015;82:88-93.

14. Rivera N, Manuel R, Alberto J, et al. Accuracy of ASGE criteria for the prediction of choledocholithiasis. Revista Espanola de Enfermedades Digestivas : Organo Oficial de La Sociedad Espanola de Patologia Digestiva. 2016;108(6):309-14.

15. ASGE Standards of Practice Committee, Buxbaum JL, et al. ASGE guideline on the role of endoscopy in the evaluation and management of choledocholithiasis. Gastrointest Endosc. 2019;89:1075-1105.e15.

16. Jagtap N, Hs Y, Tandan M, et al. Clinical utility of ESGE and ASGE guidelines for prediction of suspected choledocholithiasis in patients undergoing cholecystectomy. Endoscopy. 2020;52(7):569-73.

17. Videhult P, Sandblom G, Rudberg C, et al. Are liver function tests, pancreatitis and cholecystitis predictors of common bile duct stones? Results of a prospective, population-based, cohort study 
of 1171 patients undergoing cholecystectomy. HPB (Oxford). 2011;13:51927.

18. Magalhaes J, Rosa B, Cotter J. Endoscopic retrograde cholangiopancreatography for suspected choledocholithiasis: from guidelines to clinical practice. World J Gastrointest Endosc. 2015;16(7):128-34.

19. Rubin MIN, Thosani NC, Tanikella R, et al. Endoscopic retrograde cholangiopancreatography for suspected choledocholithiasis: testing the current guidelines. Dig Liver Dis. 2013;45(9):744-9.

20. Higgins JPT, Thomas J, Chandler J, et al. Cochrane Handbook for Systematic Reviews of Interventions version 6.2 (updated February 2021). Cochrane, 2021.

21. He H, Tan C, Wu J, et al. Accuracy of ASGE high-risk criteria in evaluation of patients with suspected common bile duct stones. Gastrointest Endosc. 2017;86:525-32.

22. Kuzu UB, Ödemiş B, Dişibeyaz S, et al. Management of suspected common bile duct stone: diagnostic yield of current guidelines. HPB (Oxford). 2017;19:126-32.

23. Panda N, Chang Y, Chokengarmwong N, et al. Gallstone pancreatitis and choledocholithiasis: using imaging and laboratory trends to predict the likelihood of persistent stones at cholangiography. World J Surg. 2018;42:3143-9.

24. Suarez AL, LaBarre NT, Cotton PB, et al. An assessment of existing risk stratification guidelines for the evaluation of patients with suspected choledocholithiasis. Surg Endosc. 2016;30:4613-8.

25. Jeon TJ, Cho JH, Kim YS, et al. Diagnostic value of endoscopic ultrasonography in symptomatic patients with high and intermediate probabilities of common bile duct stones and a negative computed tomography scan. Gut Liver. 2017;11:290-7.

26. Sethi S, Wang F, Korson AS, Krishnan S, et al. Prospective assessment of consensus criteria for evaluation of patients with suspected choledocholithiasis. Dig Endosc. 2016;28:75-82.
27. Kamath SU, Dharap SB, Kumar V. Scoring system to preoperatively predict choledocholithiasis. Indian J Gastroenterol. 2016;35:173-8.

28. Aleknaite A, Simutis G, Stanaitis J, et al. Risk assessment of choledocholithiasis prior to laparoscopic cholecystectomy and its management options. United European Gastroenterol J. 2018;6:428-38.

29. Lee HW, Song TJ, Park DH, et al. Diagnostic performance of the current risk-stratified approach with computed tomography for suspected choledocholithiasis and its options when negative finding. Hepatobiliary Pancreat Dis Int. 2019;18:366-72.

30. Chisholm PR, Patel AH, Law RJ, et al. Preoperative predictors of choledocholithiasis in patients presenting with acute calculous cholecystitis. Gastrointest Endosc. 2019;89:977-983.e2.

31. Yang MH, Chen TH, Wang SE, et al. Biochemical predictors for absence of common bile duct stones in patients undergoing laparoscopic cholecystectomy. Surg Endosc. 2008;22:1620-4.

32. Bose SM, Mazumdar A, Prakash VS, et al. Evaluation of the predictors of choledocholithiasis: comparative analysis of clinical, biochemical, radiological, radionuclear, and intraoperative parameters. Surg Today. 2001;31:117-22.

33. Chan T, Yaghoubian A, Rosing D, et al. Total bilirubin is a useful predictor of persisting common bile duct stone in gallstone pancreatitis. Am Surg. 2008;74:977-80.

34. Rahal MA, Rammal MO, Karaoui W, et al. Predictors of bile tree pathology in patients presenting with gallbladder disease. Eur J Gastroenterol Hepatol. 2017;29(9):1017-21.

Publisher's Note Springer Nature remains neutral with regard to jurisdictional claims in published maps and institutional affiliations. 